\title{
Energieträgerübergreifende Planung und Analyse von Energiesystemen
}

\author{
M. Heimberger, T. Kaufmann, C. Maier, S. Nemec-Begluk, A. Winter OVE, W. Gawlik
}

\begin{abstract}
Das gesamte Energiesystem der Strom-, Gas- und Wärmeversorgung befindet sich in einem Umbruch. Dieser Umbruch wird einerseits durch eine Veränderung in der Erzeugungsstruktur und andererseits durch ein geändertes Verbraucherverhalten getrieben. Besonders das elektrische System wird durch diese Veränderungen an seine betrieblichen Grenzen gebracht. Die Kopplung vorhandener Infrastrukturen bietet hier eine mögliche Abhilfemaßnahme, die zusätzlich zu einer besseren Ausnutzung bestehender Infrastrukturen führen kann. In diesem Artikel werden die an der Technischen Universität Wien am Institut für Energiesysteme und Elektrische Antriebe (Arbeitsgruppe Elektrische Anlagen) entwickelten Methoden zur Planung, Auslegung und dem Betrieb von Hybridnetzen ("Universal Grids") vorgestellt und Ergebnisse anhand durchgeführter Forschungsprojekte veranschaulicht.
\end{abstract}

Schlüsselwörter: Hybridnetze; systemübergreifende Energieversorgung; Energiespeicher; Energieeffizienz; Exergieeffizienz; Optimierung

\section{Planning and analysis of universal grids.}

The entire energy system of electricity-, gas- and heat-supply faces major changes. These changes are driven by evolution of the generation structure on the one hand and by changes in the consumer behavior on the other hand. Because of that, especially the electrical power system is brought to its limits. The coupling of existing energy infrastructure could be a possible solution to this problem, which could also result in a better utilization of energy networks. This paper describes methods and achieved results for planning, analyzing and operating hybrid grids ("universal grids"), developed in research projects associated with this field at the Institute of Energy Systems and Electrical Drives (ESEA) at the TU Wien.

Keywords: universal grid; multi-system energy supply; energy storage; energy efficiency; exergy efficiency; optimization

Eingegangen am 19. Jänner 2017, angenommen am 15. März 2017, online publiziert am 8. Mai 2017

(c) The Author(s) 2017. Dieser Artikel ist auf Springerlink.com mit Open Access verfügbar

\section{Einleitung}

\subsection{Motivation für Hybridnetze}

Ein erheblicher Anteil (ca. drei Viertel) des gesamten österreichischen Endenergiebedarfes wird mit fossilen Energieträgern gedeckt [1, 2]. Im Bereich Raumwärme beträgt der nicht erneuerbare Anteil ca. 59 Prozent [3], bei der Stromproduktion liegt dieser Anteil gemäßStromkennzeichnung dagegen bei ca. 13 Prozent [4]. Österreich hat sich zur Reduktion von Treibhausgasemissionen und zur Einsparung von Endenergie bekannt und seine Bestrebungen in den „2020 Zielen" festgehalten. Diese geben folgendes vor [5]:

- Gemessen am Bruttoinlandsverbrauch soll der Anteil erneuerbarer Energieträger auf 34 Prozent und am Endenergieverbrauch im Verkehrssektor auf 10 Prozent erhöht werden.

- Die Treibhausgasemissionen in Sektoren, die nicht dem Emissionshandel unterliegen, müssen um mindestens 16 Prozent gegenüber den Emissionen aus 2005 reduziert werden. Für Sektoren, die dem Emissionshandel unterliegen, gilt eine Reduktion um 21 Prozent.

- Die Energieeffizienz soll gegenüber einem Baseline-Szenario um 20 Prozent erhöht werden.

Für die Erreichung der österreichischen „2020 Ziele” ist deshalb der weitere Ausbau regenerativer Erzeuger in der Energieversorgung unumgänglich [6]. Vor allem das bestehende elektrische Verteilnetz ist auf einen massiven Ausbau dezentraler, erneuerbarer
Einspeisung (insbesondere Windkraft und Photovoltaik) nicht vorbereitet und stößt meist durch Spannungsbandverletzungen oder Betriebsmittelüberlastungen auf Probleme. Durch erneuerbare Erzeugungsanlagen können sehr große Erzeugungsspitzen aufgrund der hohen Gleichzeitigkeit in der Erzeugung auftreten, die das elektrische Energiesystem an seine Leistungsgrenzen bringen. Zudem treten die hohen Erzeugungsspitzen meistens nicht zeitgleicht mit den Lastspitzen auf, was in einem sehr volatilen Residuallastprofil resultiert. Das Residuallastprofil stellt die Differenz zwischen Erzeugung und Verbrauch dar. Hier stehen mehrere Abhilfemaßnahmen zur Verfügung: Netzausbau, Abregelung, Speicherintegration, Demand Side Management (DSM) oder Kopplung bestehender Energieinfrastrukturen. Netzausbau und Abregelung der regenerativen Einspeiser stellen derzeit diskutierte Abhilfemaßnahmen bei Netzengpässen dar. Durch Abregelung wird eine gezielte Reduktion der

Heimberger, Markus, Technische Universität Wien, Institut für Energiesysteme und Elektrische Antriebe, Gußhausstraße 25-25a/370-1, 1040 Wien, Österreich

(E-Mail: markus.heimberger@tuwien.ac.at); Kaufmann, Thomas, Technische Universität Wien, Institut für Energiesysteme und Elektrische Antriebe, Gußhausstraße 25-25a/370-1, 1040 Wien, Österreich; Maier, Christoph, Technische Universität Wien, Institut für Energiesysteme und Elektrische Antriebe, Gußhausstraße 25-25a/370-1, 1040 Wien, Österreich; Nemec-Begluk, Sabina, Technische Universität Wien, Institut für Energiesysteme und Elektrische Antriebe, Gußhausstraße 25-25a/370-1, 1040 Wien, Österreich; Winter, Alexander, Technische Universität Wien, Institut für Energiesysteme und Elektrische Antriebe, Gußhausstraße 25-25a/370-1, 1040 Wien, Österreich; Gawlik, Wolfgang, Technische Universität Wien, Institut für Energiesysteme und Elektrische Antriebe, Gußhausstraße 25-25a/370-1, 1040 Wien, Österreich 
eingespeisten Leistung erzielt. Zusätzlich kann mittels eines Einsatzes von Speicher- und Kopplungstechnologien das Residuallastprofil verändert werden. Durch koordiniertes Ein- und Ausspeichern bzw. Umwandlung in eine andere Energieform, ist somit auch die Vermeidung von Netzengpässen möglich. Eine Erhöhung des regenerativen Energieverbrauchs und die Verlagerung der "erneuerbaren Energie" in andere Energiesysteme werden damit zusätzlich gewährleistet. Die aktive Änderung bzw. Anpassung des Stromverbrauchs auf der Verbraucherseite wird in dieser Arbeit als Demand Side Management (DSM) bezeichnet. Mittels DSM kann ein Teil des Stromverbrauchs entsprechend dem Erzeugungsverlauf angepasst werden und somit eine Glättung des Residuallastprofils realisiert werden.

Abregelung ist mit Sicherheit der am einfachsten zu realisierende Ansatz und je nach Ausbaugrad der erneuerbaren Energieträger müsste auch nur ein Bruchteil der theoretisch jährlich erzeugten Energiemenge abgeregelt werden. Jedoch stellt dies keine Lösung für Erzeugungslücken dar. Elektrische Langzeitspeicher wie zum Beispiel Pumpspeicherkraftwerke sind in ihrem zusätzlichen Ausbau in Österreich oder Europa aufgrund der geographischen Anforderungen begrenzt. Und direkte elektrische Speicher in Form von Batteriespeicher sind für großtechnische Umsetzungen (einige $100 \mathrm{MW}$ und einige $100 \mathrm{MWh}$ ) im Verhältnis zu Pumpspeicherkraftwerken oder Gasspeichern in Kombination mit Power to Gas-Anlagen teurer. Einen großen Beitrag zur Integration erneuerbarer Erzeugungsanlagen, insbesondere in das Stromnetz, kann die Kopplung parallel, vorhandener Infrastrukturen (Strom-, Gas- und Fernwärmesystem) leisten. In Abb. 1 ist diese Kopplung schematisch dargestellt. Die direkte Verwendung von Strom stellt den effizientesten Ansatz dar und sollte bevorzugt werden. Ist dies jedoch nicht möglich, dann stellt die Umwandlung in andere Energieformen trotz der Umwandlungsverluste, eine gute Alternative dar. Parallele Energiesysteme zur Strom-, Gas- und Fernwärmeversorgung können dadurch besser ausgenutzt werden und elektrische Energieüberschüsse in ein anderes System übertragen werden. Des Weiteren kann auf (saisonale) Speicherpotentiale im Gas- oder Fernwärmesysteme zurückgegriffen werden, um die Herausforderung der Energiespeicherung zu unterstützen. Letztlich wird dadurch auch ein Beitrag zur Reduktion des Einsatzes nichterneuerbarer Energieträger geleistet.

\subsection{Aufbau und Ziel des Artikels}

Die Kopplung der Energiesysteme Strom, Gas und Wärme kann für sich alleine nicht als Komplettlösung für die Erreichung der Klimaziele im Bereich Strom und Wärme angesehen werden. Es wird vielmehr ein Mix mit weiteren Maßnahmen wie z. B. Abregelung und DSM notwendig sein. Dabei wird jedoch der Ansatz der Systemkopplung ein wesentlicher Bestandteil sein, ohne dem das zukünftige Energiesystem im Umfeld erneuerbarer Erzeugung seinen Anforderungen nicht gerecht werden kann. Deshalb verfolgt dieser Artikel zuerst das Ziel ein gemeinsames Verständnis von Hy bridnetzen zu schaffen und Kopplungstechnologien sowie deren Verortung im Hybridnetz darzustellen. Danach werden kombinierte Modellierungs- und Optimierungsansätze für Strom-, Gas- und Wärmenetze unter Betrachtung von Stakeholdern und weiteren Maßnahmen (Einsatz von Speichertechnologien, Abregelung, DSM) vorgestellt. Die Schlussfolgerungen werden darauf aufbauend anhand von Kernaussagen durchgeführter Forschungsprojekte am Institut für Energiesysteme und Elektrische Antriebe der TU Wien gezogen.

\subsection{Definition}

Unter dem Begriff "Hybridnetz" wird üblicherweise die Verschränkung von Strom-, Gas- und Wärmenetzen (manchmal zusätzlich auch Kältenetzen) durch Kopplungstechnologien wie Power-to-Gas, Power-to-Heat und ähnlichem verstanden, wobei nicht zwangsläufig alle genannten Energiesysteme gleichzeitig vorhanden sein müssen.

In [7] wird folgende, allgemein gültige Formulierung für "Hybridnetze" definiert:

"Unter einem Hybridnetz verstehen wir ein energiedomänenübergreifendes (oder auch intersektorielles) Energiesystem, in dem Energie jeweils in ihrer aktuellen Form verbraucht, gespeichert oder transportiert oder aber über eine Konversion in eine andere Energieform gewandelt werden kann, in der sie wiederum verbraucht, gespeichert oder transportiert werden kann."

Durch die Kopplung der Energienetze kann somit der Energieverbrauch für die betrachtete Energieform, flexibilisiert und zusätzliche Speichermöglichkeiten erschlossen werden. Insbesondere in Netzen mit einem hohen Anteil an volatilen Erzeugern kann die Verbindung der Energienetze zur Verbesserung der Netzstabilität beitragen und die bestehende Energieinfrastruktur effizienter ausgenützt werden.

Neben dem Begriff "Hybridnetz" werden weitere Bezeichnungen, wie "Polynetz" oder "Universal Grid" verwendet.

Wesentliche Bestandteile eines Hybridnetzes sind die eingesetzten Schnittstellentechnologien bzw. Kopplungstechnologien. Diese verbinden die Energienetze durch deren Möglichkeit zur Umwandlung einer Energieform in eine andere. Daneben sind Energiespeichertechnologien von wesentlicher Bedeutung. Sie ermöglichen, Energie zwischen zu speichern und zu einem späteren Zeitpunkt wieder in eines der betrachteten Energienetze abzugeben. Dafür geläufigen Technologien sind in Abb. 1 dargestellt.

Abhängig von der Netzebene (Übertragungsnetz-, Verteilnetzebene) werden folgende Technologien unterschieden:

- zentrale Kopplungstechnologien: Kopplung der übergeordneten Energienetze und anschließender Transport in untergeordnete Netzebenen. Technologien: Gasturbinen, Gas- und Dampfkraftwerke mit/ohne Fernwärmeauskopplung (GuD).

- dezentrale Kopplungstechnologien: Kopplung untergeordneter Verteilnetze oder eine Kopplung der Energienetze bzw. Energieträger lokal beim Energiekunden (Groß- und Kleinkunden). Technologien: Wärmepumpen, Brennstoffzellen, Elektrolyseure, Kraft-Wärme-Kopplungsanlagen (KWK-Anlagen), Geothermieanlagen, Gasmotoren/-turbinen, Reformatoren, Methanisierungsanlagen und (Gas-)Kessel.

- Langzeitspeicher: Diese Speicher weisen üblicherweise ein großes Energie-zu-Leistungs-Verhältnis (E/P-Verhältnis) auf. Laut [8] beträgt dieses Verhältnis ca. $720 \mathrm{~h}$. Diese Speichertypen werden überwiegend für die saisonale Energieverlagerung eingesetzt. Neben zentralen und dezentralen Gasspeichern und Pumpspeicherkraftwerken kommen hierfür auch Langzeitwärmespeicher zum Einsatz.

- Kurzzeitspeicher: Speicher mit kleinem E/P-Verhältnis werden für den kurzzeitigen Ausgleich von Energieungleichgewichten herangezogen. Im Stromnetz kommen hier insbesondere Blei- oder LiIonen-Akkumulatoren zum Einsatz mit einem E/P-Verhältnis von bis zu $4 \mathrm{~h}$ [8], im Wärme- und Gasnetz Tagesspeicher.

In Anbetracht der schon bestehenden Kopplungstechnologien wie GuD-Kraftwerke, Wärmepumpen, KWK-Anlagen, usw. kann festgehalten werden, dass Teile eines Hybridnetzes heute schon existieren. Einen besonderen Kopplungspunkt der Energienetze stellt 


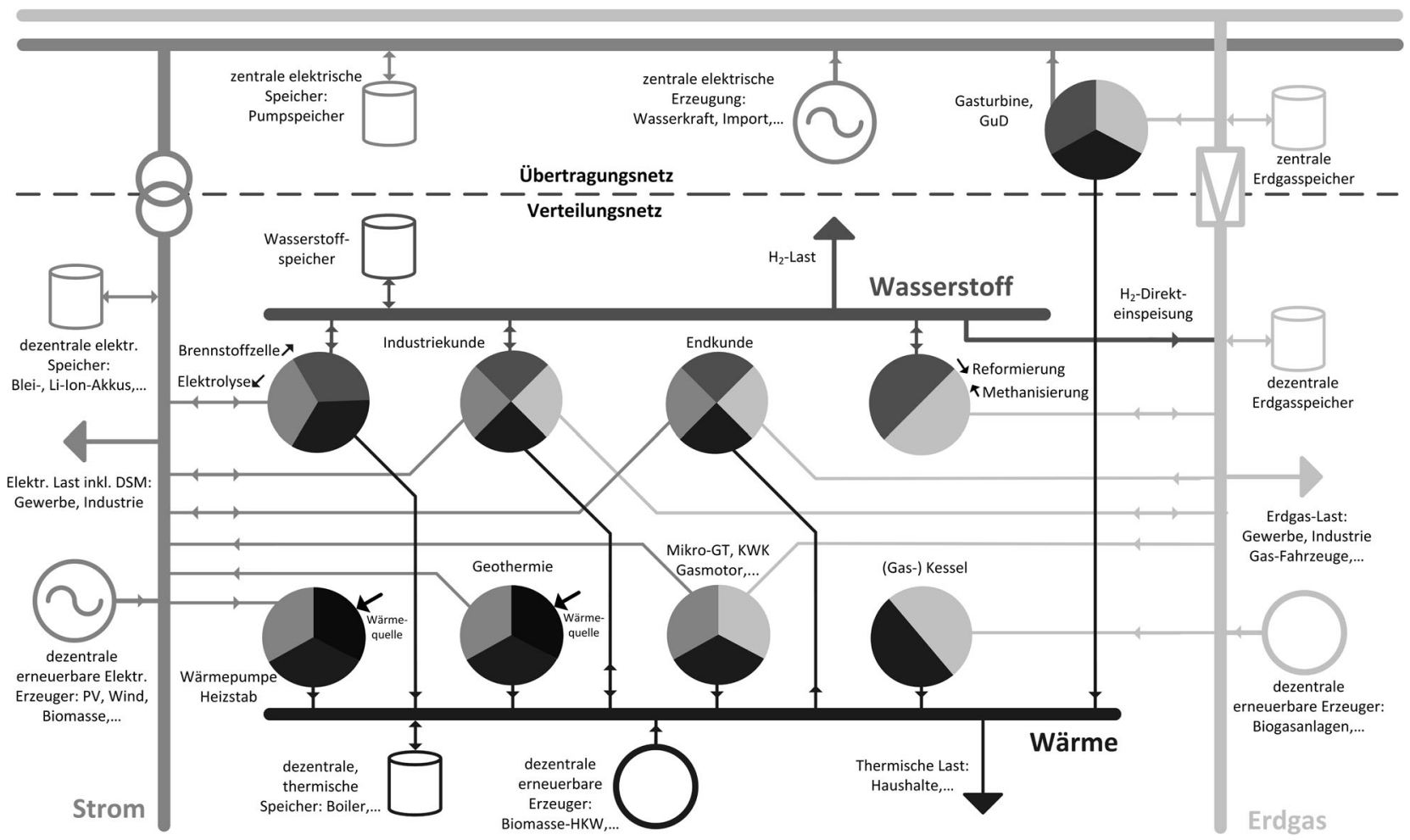

Abb. 1. Technologien zur Koppelung der Energienetze im Hybridnetz und deren Verortung in unterschiedlichen Netzebenen [Quelle: modifizierte Darstellung aus dem Projekt „OPEN HEAT GRID“]

der Mittelspannungskunde bzw. Industriekunde dar. Dieser besitzt oftmals bereits die geeigneten Anschlüsse zum Strom, Gas- und Wärmenetze und kann Umwandlungspfade zwischen allen betrachteten Energieträgern ermöglichen. Der Industriekunde kann beispielsweise über die Elektrolysetechnologie Wasserstoff produzieren und somit elektrische Energie in einen anderen Energieträger umwandeln, zwischenspeichern und verlagern. Der erzeugte Wasserstoff kann für interne Industrieprozesse eingesetzt werden oder für weitere Anwendungszwecke (z. B. Transport) ausgekoppelt werden. Auch eine Rückverstromung des Wasserstoffs ist möglich. Zusätzlich besteht auch die Möglichkeit Wasserstoff direkt in das Erdgasnetz einzuspeisen oder umgekehrt bei Schwerindustrie durch Erdgasreformierung Wasserstoff zu erzeugen und somit eine bidirektionale Kopplung von Wasserstoff und Erdgasnetz zu gewährleisten. Den Wärmebedarf kann der Industriekunde mittels direkter, elektrischer Heizung (durch Heizstäbe oder Wärmepumpen), Erdgas (im Gaskessel) oder durch Abwärmenutzung (von GuD-Anlage, KWK-Anlage oder Brennstoffzelle) decken.

Beim Kopplungspunkt "(Haushalts-)Endkunde" sind aufgrund fehlender Anschlüsse im Normalfall nicht alle möglichen Umwandlungspfade machbar und sinnvoll. Im Gegensatz zur bidirektionalen Kopplung von Haushalten mit eigener regenerativer Stromerzeugung mit dem Stromnetz, ist die Rückspeisung von dezentral erzeugter Wärme (z. B. Wärmepumpe, Blockheizkraftwerk, Solarthermie) aufgrund des lokalen Kreislaufsystems eines Wärmenetzes mit Vor- und Rücklauf mit wesentlich größeren technischen Herausforderungen verbunden. Neben fehlendem Abwärmepotenzial in Haushalten, gibt es bei Wärmenetzen in Österreich kein Unbundling zwischen Erzeugung, Netz und Vertrieb, sowie keine Abnahmeverpflichtung (bilaterale Vereinbarungen notwendig). Bei kleinen Elektrolyseanlagen für den Privatgebrauch steht normalerweise der Autarkiegedanke im Vordergrund und eine Einspeisung von dezentral erzeugtem Wasserstoff in das Erdgasniederdrucksystem ist prinzipiell technisch, als derzeit auch rechtlich nicht vorgesehen.

\section{Methodik}

\subsection{Modellierung}

In unterschiedlichen Projekten wurden am Institut für Energiesysteme und Elektrische Antriebe an der TU Wien, Hybridnetze analysiert und deren Nutzeffekte im Rahmen einer Gesamtsystemoptimierung evaluiert. Dafür wurden wesentliche Komponenten der Hybridnetze und die Verknüpfung zwischen den einzelnen Komponenten mathematisch in Form von Nebenbedingungen modelliert. Als Komponenten der Hybridnetze wurden die jeweiligen Energienetze, Kopplungstechnologien, Verbraucher, Erzeugungstechnologien, Speichertechnologien und Demand Side Management verstanden. Dieser Abschnitt behandelt die entwickelten Optimierungs- und Modellierungsansätze von Hybridnetzen.

Alle in der Arbeitsgruppe Elektrische Anlagen durchgeführten Projekte bzw. wissenschaftliche Arbeiten zum Thema Hybridnetze verfolgten das Ziel, das Konzept der Hybridnetze hinsichtlich ausgewählter Zielvorgaben optimal einzusetzen und zu betreiben. Bei diesen Untersuchungen wurden neben der Kopplung bestehender Energienetze auch andere Abhilfemaßnahmen wie Speichertechnologien und Abregelung berücksichtigt und in den Zielvorgaben integriert. Alle untersuchten Optimierungsmodelle wurden als lineare Optimierungsprobleme abgebildet. Gründe für die Wahl der linearen Programmierung waren die Größe des Optimierungsproblems, die überproportional zur Variablenanzahl ansteigt und die Tatsache, dass bei der linearen Optimierung das lokale Optimum mit dem globalen Optimum übereinstimmt. 


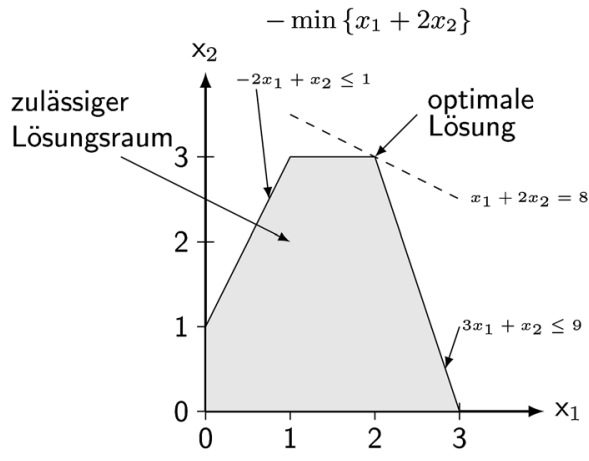

Abb. 2. Beispiel eines linearen Optimierungsproblems

Lineare Optimierungsprobleme bestehen aus $n$-Entscheidungsvariablen, $m$-Nebenbedingungen und einer Zielfunktion. Die Grundform des Optimierungsproblems in Matrix-Form lautet:

$$
\min \left\{\underline{c}^{T} \cdot \underline{x}\right\}, \quad \underline{x} \in \Re^{n \times 1} \quad \underline{c} \in \Re^{n \times 1}
$$

$$
\boldsymbol{A} \cdot \underline{x} \geq \underline{b}, \quad A \in \Re^{m \times n} \quad \underline{b} \in \Re^{m \times 1}
$$

$$
\underline{x} \geq 0
$$

Wobei der Vektor $\underline{x}$ die Entscheidungsvariablen, die Matrix $\boldsymbol{A}$ und der Vektor $\underline{b}$ die Nebenbedingungen und der Vektor $\underline{c}$ die Koeffizienten der Entscheidungsvariablen in der Zielfunktion darstellen. Das lineare Optimierungsproblem mit der Zielfunktion (1), hat die Aufgabe die Entscheidungsvariablen so zu wählen, dass unter Einhaltung der Nebenbedingungen (2) und (3), ein Minimum der Zielfunktion erzielt wird. Ein Beispiel eines linearen Optimierungsproblems mit zwei Entscheidungsvariablen und fünf Nebenbedingungen, die den Lösungsraum des Problems abgrenzen, ist graphisch in der Abb. 2 dargestellt.

In Abhängigkeit der Größe des Optimierungsproblems und der verlangten Modellierungsgenauigkeiten der Untersuchungen wurde in den durchgeführten Arbeiten zwischen der linearen Optimierung und der linearen gemischt ganzzahligen Optimierung unterschieden. Das Ziel in den Untersuchungen war es, das Energiesystem unter Einhaltung aller Nebenbedingungen durch Minimieren bzw. Maximieren der Zielfunktion zu optimieren. Die Nebenbedingungen bildeten den Betrieb der Komponenten und die Verknüpfungen der Komponenten des Hybridnetzes ab.

Grundsätzlich wurden zur Aufbereitung der Daten als Basis für die zu behandelnde Problemstellung entweder die Programmierumgebung MATLAB ${ }^{\circledR}$ oder Python ${ }^{\mathrm{TM}}$ verwendet. Zur eigentlichen Modellierung des Optimierungsproblems kamen die Toolbox YALMIP für MATLAB ${ }^{\circledR}$, das Gurobi Python Interface als auch die alleinstehende Umgebung GAMS zum Einsatz. GAMS kommunizierte dabei zur Datenübergabe mittels Schnittstellen mit MATLAB ${ }^{\circledR}$ oder Python ${ }^{\mathrm{TM}}$. Es wurden die für wissenschaftliche Zwecke frei verfügbaren Optimierer CPLEX und Gurobi zur Lösung der definierten Optimierungsprobleme verwendet.

Die Nebenbedingungen stellen neben der Zielfunktion und den Entscheidungsvariablen den zentralen Punkt in einem Optimierungsmodell dar. Die Anzahl und der Aufbau der Nebenbedingungen beschränken den für die Optimierung zulässigen Lösungsraum. Im Folgenden werden die Modellierungsansätze der einzelnen Komponenten, welche in Form von Nebenbedingungen erfolgte, dargestellt.
Energienetze bestehen aus Knoten, die über Zweige (wie z. B. Leitungen, Kabel, Rohre) miteinander verbunden sind und somit eine zusammenhängende Netzstruktur darstellen. Dementsprechend sind der Transport und die Verteilung der Energie leitungsgebunden. Die Topologie der einzelnen Netzknoten wurde im Rahmen der Untersuchungen für jedes Energienetz über eine Knoten-ZweigInzidenzmatrix $\boldsymbol{A}$ der Dimension $n_{k} \times n_{l}\left(n_{k} \ldots\right.$ Anzahl Netzknoten, $n_{l} \ldots$ Anzahl der Netzzweige) abgebildet.

$\boldsymbol{A}=\left\{\begin{array}{l}+1, \text { wenn der Zweig I vom Knoten } k \text { wegführt } \\ -1, \text { wenn der Zweig I zum Knoten } k \text { hinführt } \\ 0, \text { wenn der Zweig I mit dem Knoten } k \text { nicht verbunden ist }\end{array}\right.$

Die Verknüpfung der einzelnen Energieträgernetze erfolgt an Standorten an denen mehrere Energieträger vorhanden sind, über die Definition eines Energieknotens. Dieser ermöglicht über die Definition einer Kopplungsmatrix, besetzt mit den Wirkungsgraden der verwendeten Umwandlungstechnologien, die Beschreibung eines energieträgerübergreifenden Gesamtsystems (Abb. 1). Der Transport und die Verteilung der Energie in den einzelnen Energieträgernetzen hängen neben dem topologischen Aufbau von der Lastsituation, den Parametern der Betriebsmittel und dem Netzbetrieb ab. Die Berechnung des Energieflusses und die daraus resultierende Analyse der jeweiligen Netze wird gegenwärtig mit Hilfe konventioneller Netzsimulationssoftware (z. B. PSS ${ }^{\circledR}$ SINCAL, NEPLAN ${ }^{\circledR}$, DIgSILENT) durchgeführt. Die hinterlegten Berechnungsmethoden umfassen jedoch nichtlineare Gleichungen, die in einem iterativen Prozess das Ergebnis der Lastverteilung auf den Zweigen und die damit verbundenen Auswirkungen auf das Netz liefern. Im Stromnetz werden solche Lösungen mit klassischen $A C^{1}$ Lastflussberechnungen und für die Strömungsberechnungen im Gasnetz mit dem Hardy-Cross Verfahren ermittelt. Iterative und nichtlineare Berechnungsmethoden können im Rahmen der linearen Programmierung nicht durchgeführt werden. Dementsprechend wurden vereinfachte Berechnungsmethoden für die Abbildung der Energieverteilung in den Energienetzen angewendet.

Elektrisches Netz: Im elektrischen System wurde für die Lastflussberechnung die reine Wirklastflussberechnung bzw. DC-Lastflussrechnung ( $D C=$ Direct Current) herangezogen. Dadurch wird die nichtlineare Wirkleistungsberechnung linearisiert. Durch die DCLastflussrechnung wird die Lastaufteilung auf die einzelnen Leitungen basierend auf den Wirkleistungseinspeisungen und Wirkleistungsverbräuchen in den einzelnen Netzknoten berechnet. Der Blindleistungstransport wird dabei aber vernachlässigt. Zusätzlich können keine Aussagen über die auftretenden Spannungswerte in den Netzknoten getroffen werden. Zudem sind die stromabhängigen Leitungsverluste nur näherungsweise bestimmbar.

In vereinfachter Form kann folgender Zusammenhang (4) zwischen den Knotenwirkleistungen $P_{i}$ ( $i$ bzw. $j$ bezeichnen die Laufvariablen der Netzknoten) und den Winkeln der Knotenspannungen $\vartheta$ (Spannungswinkeldifferenz zwischen dem "Slack-Knoten" bzw. Referenzknoten und Netzknoten $j$ ) über den Imaginärteil der Knotenpunktsadmittanzmatrix $Y$ und der Nennspanung $U_{n}$ hergestellt werden:

$$
P_{i}=-U_{n}^{2} \sum_{j=1}^{n_{k}} \Im\left\{Y_{i j}\right\} \cdot \vartheta_{j}
$$

\footnotetext{
${ }^{1} \mathrm{AC}$ : engl. Alternate Current $=$ Wechselstrom
} 
(5) beschreibt diesen Zusammenhang in Matrixschreibweise. Für Betriebsmittel, wie Freileitungen die ein verhältnismäßig kleines $R / X$ Verhältnis aufweisen, können die Zweigadmittanz auf die Imaginärteile bzw. auf die Knotenpunktsuszeptanzmatrix $\boldsymbol{B}$, die die Dimension $n_{k} \times n_{k}$ aufweist, reduziert werden.

$$
\boldsymbol{p}=-U_{n}^{2} \cdot \boldsymbol{B} \cdot \boldsymbol{\vartheta}
$$

Durch Elimination des "Slack-Knotens" in den Matrizen $\boldsymbol{A}$ und $\boldsymbol{B}$ und dem Einführen der daraus reduzierten Knoten-Zweig-Inzidenzmatrix $\boldsymbol{A}^{\prime}$ und der reduzierten Knotenpunktsuszeptanzmatrix $\boldsymbol{B}^{\prime}$, die den Zusammenhang der Winkeldifferenzen zwischen Anfang und Ende eines Leitungsabschnittes beschreiben, kann die Zweigbelastung bestimmt werden (6). Dabei werden aus den Winkeln der Knotenspannungen und deren Multiplikation mit der Zweigsuszeptanzmatrix $\boldsymbol{B}_{\boldsymbol{I}}$ die Zweigflüsse zwischen zwei Netzknoten $i$ und $j$ wie folgt bestimmt:

$$
P_{i j}=-U_{n}^{2} \Im\left\{Y_{i j}\right\} \cdot\left(\vartheta_{i}-\vartheta_{j}\right)
$$

Der vollständige Zusammenhang zwischen den bekannten Knotenleistungen $\boldsymbol{p}$ und den resultierenden Zweigbelastungen $\boldsymbol{p}_{l}$ ist in (7) dargestellt. Dabei werden alle Größen, die um den Referenzknoten reduziert wurden mit ' gekennzeichnet.

$$
\boldsymbol{p}_{l}=\boldsymbol{B}_{/} \cdot \boldsymbol{A}^{\prime} \cdot \boldsymbol{B}^{\prime-1} \cdot \boldsymbol{p}^{\prime}
$$

Das Matrizenprodukt $\boldsymbol{B}_{\mid} \cdot \boldsymbol{A}^{\prime} \cdot \boldsymbol{B}^{-1}$ wird ausschließlich aus der Topologie und den Parametern des Stromnetzes bestimmt und bleibt für ein nicht verändertes Netz konstant.

Im Rahmen der Optimierung wurde die oben definierte Gleichung für die Zweigbelastung zuzüglich der zulässigen Grenzen für die Auslastungen der Betriebsmittel als Nebenbedingung hinterlegt.

Gas- und Wärmenetze: Aus den Berechnungen der stationären hydraulischen Zustände ermitteln sich die Systemdrücke an den Abnehmern und Knotenpunkten sowie die Strömungsgeschwindigkeiten in den Rohrleitungen im Netzabschnitt. Um diese Berechnungen als Nebenbedingung in die Optimierung zu integrieren, muss eine Linearisierung des Systems durchgeführt werden. Grundsätzlich ist der Druckabfall an einer Leitung über die Gleichung von DarcyWeisbach (8) definiert.

$$
\Delta p=\lambda \frac{8 / \rho}{d^{5} \pi^{2}} \dot{V^{2}}
$$

Dabei beschreibt $\rho$ die Dichte des Energieträgers Gas, $d$ den Durchmesser und / die Länge der Rohrleitung. Unter der Annahme einer konstanten Rohrreibungszahl $\lambda$ im betrachteten Netzbereich erfordert eine lineare Berechnung der hydraulischen Zustände die Linearisierung des quadratischen Zusammenhanges, angeführt in (9), zwischen dem Druckverlust $\Delta p$ an einer Leitung und dem Volumenstrom $\dot{V}$ in den Rohren.

$$
\Delta p=f\left(\dot{V}^{2}\right)
$$

Dieser Zusammenhang wurde im Modell durch eine stückweise lineare Approximation der quadratischen Abhängigkeit abgebildet. Die Auswirkungen auf die Genauigkeit der Berechnungsergebnisse durch die getroffenen Annahmen wurden in [9] behandelt. Es zeigte sich, dass für Niederdruckgebiete mit hohen Lastdichten und der daraus folgenden geringen Leitungslängen zwischen den Abnahmeknoten keine signifikanten Auswirkungen auf die Berechnungsergebnisse entstehen.

Die Berechnung der Zustände im Wärmenetze erfolgt analog zu den hydraulischen Berechnungen des Gasnetzes, die durch die thermischen Berechnungen erweitert werden. Dafür wird die Temperaturdifferenz zwischen Vor- und Rücklauf $\Delta T$ am Verbraucher bestimmt, um mit der mittleren spezifischen Wärmekapazität $\mathrm{C}_{\mathrm{H} 2 \mathrm{O}}$ und

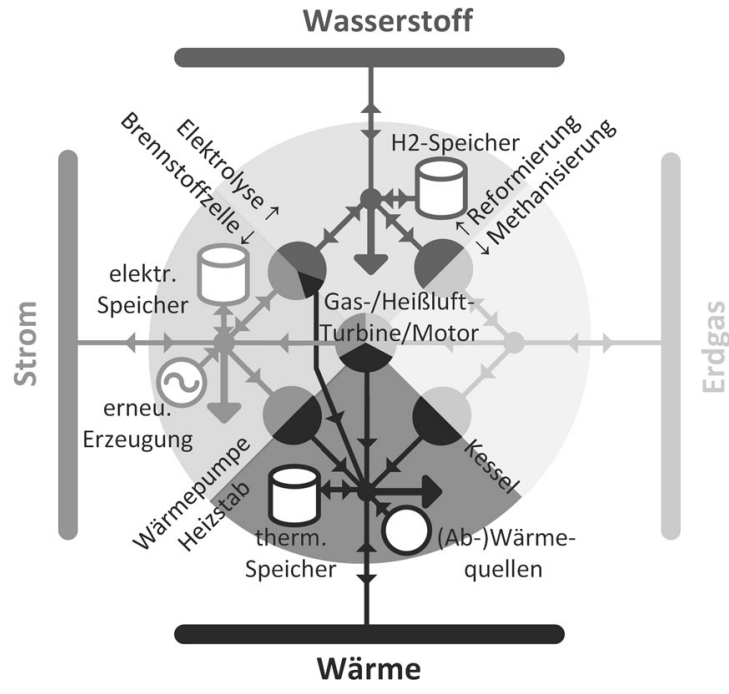

Abb. 3. Kopplung der Netze in einem Knotenpunkt [Quelle: Projekt „OPEN HEAT GRID“]

der Dichte $\rho_{H 2 O}$ des Energieträgermediums Wasser zwischen der nachgefragten Wärmeleistung $P$ des Verbrauchers und dem dafür notwendigen Volumenstrom $\dot{V}$ umzurechnen. Die Formel zur Berechnung der Wärmeleistung ist in (10) angeführt.

$$
P=\Delta T \cdot \rho_{H 2 O} \cdot C_{H 2 O} \cdot \dot{V}
$$

Nach Bestimmung des Volumenstroms wird eine hydraulische Berechnung zur Bestimmung der Drücke und Flüsse im Netz durchgeführt. Wärmeverluste in den Rohrleitungen wurden auf Grund der in den Modellnetzen geringen Auswirkungen nicht berücksichtigt.

Die minimalen erlaubten Knotendrücke sowie die maximal zulässigen Strömungsgeschwindigkeiten wurden in den Nebenbedingungen als Grenzwerte für die stationären hydraulischen Zustände der Gas- oder Fernwärmenetze berücksichtigt.

Energiebilanzierung: Für alle drei Energienetze gilt das 1. Kirchhoffsche Gesetz, das als Bilanznebenbedingung modelliert wurde. In jedem Knoten gleicht die Summe aller abfließenden Ströme der Summe aller zufließenden Ströme bzw. jeder Knoten stellt eine ausgeglichene Massenbilanz dar. Die Verknüpfung einzelner Energienetze wurde über diese Bilanzbedingungen sichergestellt in dem der abfließende Strom eines Energienetzes über Kopplungstechnologien in den zufließenden Strom des zu koppelnden Energienetzes umgewandelt wird. Dazu werden die Wirkungsgrade der Umwandlungstechnologien in der Kopplungsmatrix des weiter oben erklärten Energieknotens eingetragen.

Kopplungstechnologien: Die Modellierung der Kopplungskomponenten umfasst die Abbildung des Umwandlungsvorgangs. Dabei wurde die eingehende Energiegröße in die Kopplungstechnologie (z. B. beim Elektrolyseur Strom) als Entscheidungsvariable modelliert, die über einen Wirkungsgrad in die ausgehende EnergiegröBe durch die Kopplungstechnologie umgewandelt wird (z. B. beim Elektrolyseur Wasserstoff). Die Forderung bezüglich Einhaltung gewisser Leistungsgrenzen der Energiewandlung wurde je nach Kopplungstechnologie und Anforderung in Form von Nebenbedingungen modelliert.

In Abb. 3 ist ein Beispiel eines Koppelpunktes aus Abb. 1 detailliert abgebildet, in dem die drei Netze Gas, Wärme und das elektrische Netz mit verschiedenen Umwandlungstechnologien verbunden 


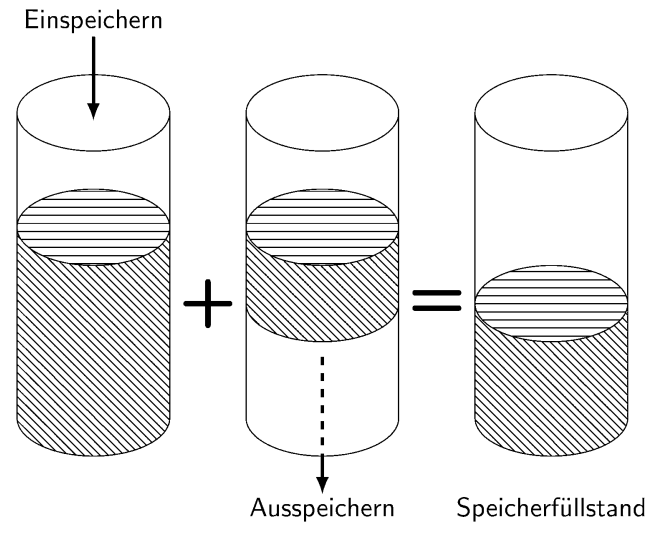

Abb. 4. Darstellung der getrennten Modellierung des Ein- und Ausspeichervorgangs

werden. Die Technologien sind entsprechend ihrer Umwandlungsrichtung in der Grafik eingetragen.

Speichertechnologien: Die Modellierung der Speicherkomponente beinhaltet das Abbilden der Speicherkapazität bzw. des Energieinhaltes und des Einspeicher- und Ausspeichervorganges der Speichertechnologie. Im Rahmen einiger Projekte und wissenschaftlicher Arbeiten hat sich die Modellierung aller drei Aspekte über den momentanen Energieinhalt $E(t)$ der Speichervorgänge bewährt. Diese Methode wurde aus der Dissertation in [10] herangezogen. Dabei wurden der Ein- und Ausspeichervorgang separat als eigenständige Speicher, die nur in eine Richtung arbeiten, abgebildet. Die Summe aus dem Ein- und Ausspeichervorgang bildet den aktuellen Energieinhalt der Speichertechnologie ab, dies ist in (11) bzw. Abb. 4 ersichtlich.

$$
0 \leq E(t)=E_{\text {aus }}(t)+E_{\text {ein }}(t) \leq E_{\text {inst }}
$$

Über die zeitliche Änderungsrate des Energieinhalts der einzelnen Speichervorgänge wurden die Lade- und Entladeleistung der Speichertechnologien modelliert. Die beiden Gl. (12a), (12b) stellen die Klemmenleistung der beiden Vorgänge dar.

$$
\begin{aligned}
& -P_{\text {aus }}(t) \cdot T=\eta_{\text {aus }} \cdot\left[E_{\text {aus }}(t)-E_{\text {aus }}(t-1)\right] \\
& -P_{\text {ein }}(t) \cdot T \cdot \eta_{\text {ein }}=\left[E_{\text {ein }}(t)-E_{\text {ein }}(t-1)\right]
\end{aligned}
$$

Die Größe $T$ stellt die Zeitauflösung der Simulation dar. Die Differenz der gesamten eingespeicherten und ausgespeicherten Energie aus (12a) und (12b) stellte die Speicherverluste dar, die in der Zielfunktion berücksichtigt werden

Im Optimierungsmodell wurden als Nebenbedingungen die Forderungen gestellt, dass die Momentanleistungen der Speichervorgänge zu jedem Zeitpunkt kleiner gleich der installierten Speicherleistung und je nach Speichervorgang größer oder gleich Null sein müssen (13a), (13b). Zudem wurde auch verlangt, dass der momentane Speicherinhalt größer gleich Null und kleiner gleich dem installierten Energieinhalt sein muss.

$$
\begin{array}{ll}
P_{\text {aus }}(t) \geq 0, & P_{\text {aus }}(t) \leq P_{\text {inst }} \\
P_{\text {ein }}(t) \leq 0, & -P_{\text {ein }}(t) \leq P_{\text {inst }}
\end{array}
$$

Demand Side Management: Die aktive Änderung bzw. Anpassung des Verbrauchs wird in dieser Arbeit als Demand Side Management (DSM) bezeichnet. Unter DSM wird hier eine Reduktion oder Erhöhung des Verbrauchs zum gewählten Zeitpunkt verstanden, der zu einem späteren Zeitpunkt durch eine Erhöhung bzw. Reduktion des Verbrauchs rückgängig gemacht wird. Dies hat keine Auswirkung auf die gesamte verbrauchte Energiemenge, sehr wohl jedoch auf den Lastgang des Verbrauches.

Grundsätzlich kann festgehalten werden, dass der Vorgang der Lastverschiebung mit einer Entladung oder Beladung eines verlustlosen Speichers gleichgestellt werden kann. Die ein- bzw. ausgespeicherte Energie muss allerdings spätestens während der maximalen erlaubten Verlagerungszeit wieder aus- bzw. eingespeichert werden. Demzufolge wurde die DSM-Modellierung mit einem verlustlosen Speichermodell durchgeführt. Der Einspeichervorgang entspricht einer Lastreduktion. Der Ausspeichervorgang ist mit Lasterhöhung gleich zu stellen.

Die Höhe der aus- und eingespeicherten Leistung der DSMMaßnahme hängt von der erlaubten Verlagerungsleistung ab. Diese Verlagerungsleistung ersetzt in der Leistungs-Nebenbedingung der Speicherkomponente die installierte Speicherleistung. Dass die verlagerte Energiemenge spätestens nach der Verlagerungszeit dem System wieder bereitgestellt sein muss, stellt eine weitere Nebenbedingung dar. Die entsprechenden Nebenbedingungen sind in (14a) und (14b) ersichtlich

$$
\begin{aligned}
& E_{\text {aus }}(t) \geq E_{\text {ein }}\left(t-t_{\text {ver }}\right) \\
& E_{\text {ein }}(t) \geq E_{\text {aus }}\left(t-t_{\text {ver }}\right)
\end{aligned}
$$

Durch die erste Nebenbedingung (14a) wird sichergestellt, dass die gesamte eingespeicherte Energie bzw. die Energiereduktion der Verbraucherlast vom Zeitpunkt $t-t_{\text {ver }}$ spätestens zum Zeitpunkt $t_{\text {ver }}$ gleich der gesamten ausgespeicherten Energiemenge bzw. der Energiemenge der Lasterhöhung ist. Die zweite Nebenbedingung (14b) bildet genau den umgekehrten Betriebsfall ab. Durch folgende Nebenbedingung

$$
-E_{\text {aus }}(N)+E_{\text {ein }}(N)=0,
$$

wird sichergestellt, dass der Energieinhalt des Speichers am Ende eines zeitlichen Horizonts $N$ Null ist und somit die gesamte konsumierte Energiemenge über diesen zeitlichen Horizont gleich bleibt. In durchgeführten Arbeiten wurde der zeitliche Horizont $N$ mit einem Tag gleichgesetzt.

\subsection{Optimierungsansätze}

Im Rahmen von Forschungsprojekten wurden ökonomische, technische und exergetische Zielvorgaben untersucht. Die einzelnen Zielfunktionen werden folgende am Beispiel der durchgeführten Projekte vorgestellt.

Im abgeschlossenen Projekt "Systemübergreifende optimale dezentrale Hybridspeicher" (Symbiose) lag das Optimierungsziel (die Zielfunktion der linearen Optimierung) in der Minimierung der Gesamtsystemkosten unter Einhaltung technischer Grenzen [11]. Je nach betrachteter Zielgruppe wurde unter Gesamtsystem die gesamte untersuchte Modellregion bzw. die Summe der einzelnen Haushalte in der Modellregion verstanden. Den Zielfunktionswert beeinflussten und veränderten die Installationskosten der neuen Technologien (dezentrale Speicher und Umwandlungstechnologien), Verlustkosten der Speicherung, Umwandlung, des Energietransports und Abregelung und Kosten bzw. Erlöse für den Energieaustausch in der Modellregion. Das gleiche Optimierungsproblem wurde für zwei Modellregionen, rurale und urbane Modellregion und unterschiedliche Zielgruppen: Netzbetreiber, Haushaltskunde, Region und bezugsminimale Region betrachtet. Für den Stakeholder Netzbetreiber wurde der Einsatz der dezentralen Speicher- und Umwandlungseinheiten untersucht, um technische Grenzen im elektrischen Netz einzuhalten und Netzverluste zu reduzieren. Das Interesse der Haushaltskunden war es, ihren Eigenverbrauch mög- 
lichst zu maximieren und in dieser Hinsicht auf dezentrale Speichertechnologien und Umwandlungseinheiten zurückzugreifen. Durch den gegebenen Preisunterschied zwischen Einspeisevergütung und Kosten für bezogene elektrische Energie wurden Haushaltskunden motiviert ihren Eigenverbrauch zu erhöhen. Aus Sicht einer Region wurde die Zielsetzung von minimalen Gesamtsystemkosten (Installationskosten und Energiekosten) verfolgt. Die Zielgruppe bezugsminimale Region wurde durch eine Erhöhung der angesetzten Energiebezugskosten des Stakeholders Region realisiert. Auf diese Weise wurde das Gesamtsystem angeregt erstmals die gesamte eigene Erzeugung innerhalb der Modellregion mit ev. Unterstützung von dezentralen Speicher- und Umwandlungseinheiten zu nutzen [11].

Die Betrachtung von energieträgerübergreifenden Zusammenhängen innerhalb des Doktoratskollegs URBEM verfolgte ein rein technisches Optimierungsziel, weshalb keine ökonomischen Auswirkungen in der Zielfunktion betrachtet werden. Es erfolgt die Minimierung der Betriebsmittelauslastungen in den Energienetzen als auch die Maximierung des Eigenverbrauchsanteils in einem festgelegten Abschnitt. Zusätzlich wird abhängig vom übergeordneten Optimierungsziel versucht, die Bezugsleistungen aus definierten Energieträgernetzen zu minimieren. Damit lassen sich technische Auswirkungen durch Rückbau eines Energieträgers (z. B. Gas) in Gebieten mit mehreren vorhandenen Energieträgernetzen ermitteln

Im Zuge des Projektes "Smart Exergy Leoben" ([12]) wurde ein exergetisches Optimierungsziel verfolgt. Dieses ist ebenfalls ein technisches Optimierungsziel, bei dem jedoch nicht ein energetisches, sondern ein exergetisches Optimum angestrebt wird. Energie kann nach dem ersten thermodynamischen Gesetz nicht verbraucht, sondern nur umgewandelt werden. Trotzdem lässt sich Energie in Form von Wärme nur bis zu einem gewissen Grad in Arbeit umwandeln. Man spricht dann von Exergie, dem nutzbaren Anteil von Wärmeenergie und Anergie, dem nicht nutzbaren Anteil von Energie. Der Zusammenhang zwischen Energie, Exergie und Anergie ist in (15) dargestellt.

$$
\text { Energie }=\text { Exergie }+ \text { Anergie }
$$

Durch eine Betrachtung des Exergiestromes anstelle des Energiestromes lassen sich somit Aussagen über die Energiequalität treffen. Eine Optimierung der Exergie der Wärme entspricht folglich einer Optimierung der Energiequalität im System. Die Exergie berechnet sich gemäß (16) aus der Energie und dem Carnotwirkungsgrad, der aus der oberen und unteren Prozesstemperatur bestimmt wird.

$$
\dot{E}=\dot{Q} \cdot \eta_{\text {Carnot }}
$$

Bei elektrischer Energie und Gas ist der Carnotwirkungsgrad gleich 1 , da physikalisch gesehen $100 \%$ der Energie dieser beiden Formen in Arbeit umgewandelt werden kann (Wirkungsgrade der Umwandlungstechnologien sind technische Restriktionen). Aus dem Quotienten der zufließenden und der abfließenden Exergie für jeden Knoten und jede Kopplungstechnologie lässt sich ein Exergiewirkungsgrad (17) berechnen, der in die Zielfunktion der Optimierung integriert werden kann.

$$
\eta_{\text {ex }}=\frac{P_{\text {ex, out }}}{P_{\text {ex }, \text { in }}}
$$

Dadurch werden für Prozesse mit hohem Exergieverlust vorwiegend Energieformen mit bereits geringem Exergieanteil verwendet, da der Verlust der Umwandlung gering bleibt.

\section{Ergebnisse}

Die Optimierungsergebnisse zeigen, dass durch die Kopplung bestehender Energieinfrastrukturen auf Verbraucherseite eine deutliche Reduktion des Gesamtenergiebezugs aus den übergeordneten Netzebenen ermöglicht werden kann. Die implementierten Kosten und Erlöse, welche beim Import und Export von Strom, Gas und Wärme erzielt wurden, spielten dabei eine entscheidende Rolle. Die Ergebnisse zeigen, dass die verbraucherseitige Kopplung von Strom und Wärme für den Stakeholder bezugsminimale Region (100-prozentiger eigener Verbrauch der innerhalb der Region erneuerbar produzierten Energie) ein wichtiger Bestandteil der Energieversorgung ist. Durch die Kopplung dezentraler paralleler Energiesysteme werden die Energieautonomie und der Eigenverbrauchsgrad einer Region stark erhöht. Eine verbraucherseitige Kopplung von Strom und Wärme ermöglicht auch eine wesentliche $\mathrm{CO}_{2}$ Emissionsreduktion, die in Abhängigkeit vom betrachteten Szenario und Region eine Bandbreite von $5 \%-82 \%$ abdecken [11].

Die Untersuchungen ergeben, dass meist die elektrische Leitungsbelastung im urbanen Umfeld und die Einhaltung des elektrischen Spannungsbandes im ruralen Gebiet die entscheidenden technischen Grenzen im Netz darstellen, aufgrund derer ein Speichereinsatz beim massiven Ausbau erneuerbarer Energieträger ohne sonstige Maßnahmen (Netzausbau, Abregelung, usw.) notwendig ist. Der Einsatz von dezentral verteilten Speichern erlaubt einen 100prozentigen Ausbau des regenerativen Erzeugungspotenzials und trägt zu einem sicheren Netzbetrieb bei. Die notwendige Speichergröße und -position ist in diesem Fall von regionalen Netzstrukturen und dem regenerativen Dargebot abhängig. Die Optimierungsergebnisse zeigen aber, dass sich unabhängig von verschiedenen Interessensgruppen (Netzbetreiber, Haushalt und gesamte Region) eine ähnliche Dimensionierung und teilweise auch ähnliche Betriebsweise der Speicher ergibt. Es werden vorwiegend dezentrale elektrische Speicher eingesetzt. Das bedeutet, die Interessen der Netzbetreiber, der Haushalte und der Region sind nicht konkurrierend, sie haben positive Effekte für alle Stakeholder. Auch bei der Installation der Wärmepumpen kann ein ähnlicher Trend erkannt werden. Einzig die Realisierung einer bezugsminimalen Region bewirkt eine stark veränderte Speicherdimensionierung, Speicherverortung und Speicherbetrieb. In diesem Fall werden die Speicher so dimensioniert, dass jegliche in der Region erzeugte Energie auch innerhalb der Region verwertet wird und es zu keiner Zeit zu einem Energieexport kommt.

Die durchgeführten Arbeiten zeigen, dass unabhängig davon ob sich eine abgegrenzte Region energetisch selbst versorgen könnte und durch welche Maßnahmen (Speicher, Kopplungstechnologien oder beides) ein vermehrter Ausbau und Nutzung erneuerbarer Energien innerhalb dieser Region möglich wäre, ein Austausch mit dem übergeordneten Netz immer anzustreben ist. Der Austausch mit Erzeugungsanlagen im übergeordneten Netz ist sowohl aus technischer als auch ökonomischer Sicht sinnvoll. Dadurch kann einerseits die benötigte Speichergröße oder das Ausmaß an Umwandlungstechnologien in realistischen Größenordnungen gehalten werden, andererseits können Synergien, in der Erzeugung und im Lastausgleich mit anderen Gebieten genutzt werden.

Aus der Sicht eines rein technischen Optimierungszieles liefern die durchgeführten Analysen Aussagen über die technisch optimale energetische Versorgung eines zukünftig entstehenden Stadterweiterungsgebietes. Durch die Verwendung einer energieträgerübergreifenden Modellierung können unterschiedliche Versorgungsmöglichkeiten anhand verschiedener Netzausbauvarianten und un- 
ter der Berücksichtigung szenarienabhängiger Laständerungen sowie des Ausbaues von Speichern und Photovoltaik- bzw. Solarthermieanlagen technisch bewertet werden. Die Optimierung bestimmt anhand der im Stadterweiterungsgebiet vorhandenen Energieträgernetze und deren Potentialen zur Leistungsaufnahme die aus technischer Sicht einsetzbaren Energieträger. Dabei ist bei gut ausgebauten Stromnetzen eine energetische Versorgung des Erweiterungsgebietes nur aus dem Stromnetz mit der Verwendung von Wärmepumpen erreichbar. In Kombination mit Photovoltaikanlagen und thermischen Speichern ist eine rein elektrische Versorgung auch für weniger gut ausgebaute Stromnetze möglich. Zusätzlich maximiert sich dadurch der Eigenverbrauchsanteil in einem Gebäudeblock. Wird durch die Einspeisung von erneuerbaren Energieträgern ein Grenzwert der Energieträgernetze verletzt, werden in der Optimierung Abregelmechanismen aktiv und ermöglichen dadurch den Betrieb der vorgegebenen AnlagengröBen.

Das primäre Ziel der exergetischen Optimierung ist es, anfallende Abwärme bzw. Verlustenergie weiter zu nutzen und somit den Primärenergiebedarf des Gesamtsystems zu verringern. Die Wärme für das Fernwärmenetz wird aus Abwärme von zum Beispiel industriellen Prozessen gewonnen, wodurch diese Energie weiter genutzt wird, anstatt Primärenergie zur Wärmeerzeugung zu verwenden. Die Ergebnisse der exergetischen Optimierung zeigen, dass eine Nutzung von Fernwärme zur Deckung des Heizbedarfs von Räumen (niedriges Temperaturniveau) sinnvoller ist als mit Gasheizungen, elektrischen Heizungen oder Wärmepumpen. Die technische Begründung ist, dass aus Raumwärme nur wenig Energie in Arbeit umgewandelt werden kann und sie somit einen geringen exergetischen Anteil besitzt. Da Fernwärme bereits einen weit geringeren Exergieanteil (je nach Vorlauftemperatur) als Gas oder elektrischer Strom hat, sind die Umwandlungsverluste in die exergetisch minderwertige Raumwärme geringer. Durch die Verwendung von Fernwärme kann der Primärenergieanteil gesenkt werden. Unter bestimmten Umständen ist es ist ebenfalls möglich, dass die Verwendung von Wärmepumpen exergetisch sinnvoller wird als Fernwärme. Dafür wird aber ein ganzjährig hoher COP (Coefficient Of Performance, Quotient aus der erzeugten Wärmeenergie und der aufgewendeten elektrischen Energie) der Wärmepumpe in der Größenordnung von 5 verlangt. Dieser Wert hängt jedoch stark von der Vorlauftemperatur im Fernwärmenetz $a b$, die sich üblicherweise ihrerseits mit der Jahreszeit ändert.

\section{Schlussfolgerungen}

Die massive Integration von erneuerbaren Erzeugern in Mittel- und Niederspannungsnetzen ist nur durch zusätzliche Maßnahmen möglich. Dabei ermöglichen ein sinnvoller Speichereinsatz und die Koppelung bestehender Energieinfrastrukturen eine deutliche Erhöhung der Nachhaltigkeit in der Energieversorgung. Die Kopplung bestehender Energieinfrastrukturen auf Verbraucherseite, vorwiegend zwischen Strom- und Wärmesystem, führt zur Reduktion des Gesamtenergiebezugs aus übergeordneten Netzebenen. Dadurch wird auch eine erhebliche Reduktion der $\mathrm{CO}_{2}$-Emissionen erreicht. Allerdings ist ein komplett dezentrales Energiesystem sowohl wirtschaftlich als auch technisch nicht zweckmäßig. Ein Austausch mit dem übergeordneten Netz ist immer sinnvoll. Durch den Speichereinsatz kann die Nutzung des regenerativen Erzeugungspotenzials in den bestehenden Netzen erhöht und ein sicherer Betrieb der bestehenden Netze gewährleistet werden. Der Einsatz von dezentralen Speichern im Hybridnetz ist sowohl für Netzbetreiber als auch für dezentrale Prosumer von Vorteil. Im Gegensatz zur klassischen energetischen Optimierung können exergetische Betrachtungen durch die Bewertung der Energiequalität zu anderen Ergebnissen führen und Wärmequellen zur Raumwärmebereitstellung bevorzugen, die einen geringeren exergetischen Anteil haben (z. B. Fernwärme). Die Verwendung von technischen Hybridnetzbetrachtungen in strategischen Planungsprozessen ermöglicht eine effizientere Gestaltung der Energienetze und eine Erhöhung der nachhaltigen Versorgungsmöglichkeiten innerhalb von Stadterweiterungsgebieten.

\section{Danksagung}

Open access funding provided by TU Wien (TUW). Das Projekt Symbiose wird aus den Mitteln des Klima- und Energiefonds gefördert und im Rahmen des Programms „NEUE ENERGIEN 2020" durchgeführt.

Das Projekt Smart Exergy Leoben wird aus den Mitteln des Klimaund Energiefonds gefördert und im Rahmen des Programms "Smart Cities Demo" durchgeführt.

Das Projekt URBEM ist ein aus den Mitteln des FTI-Fonds der Wiener Stadtwerke Holding AG mitfinanziertes Doktoratskolleg.

Stadt der Zukunft ist ein Forschungs- und Technologieprogramm des Bundesministeriums für Verkehr, Innovation und Technologie (BMVIT). Es wird im Auftrag des BMVIT von der Österreichischen Forschungsförderungsgesellschaft gemeinsam mit der Austria Wirtschaftsservice Gesellschaft mbH und der Österreichischen Gesellschaft für Umwelt und Technik ÖGUT abgewickelt.

Open Access This article is distributed under the terms of the Creative Commons Attribution 4.0 International License (http://creativecommons.org/ licenses/by/4.0/), which permits unrestricted use, distribution, and reproduction in any medium, provided you give appropriate credit to the original author(s) and the source, provide a link to the Creative Commons license, and indicate if changes were made.

\section{Literatur}

1. Statisitk Austria, Energiestatistik: Energiebilanzen österreich 1970 bis 2010, Statisitk Austria, [Online]. Available: http://www.statistik.at/web de/statistiken/ energie_und_umwelt/energie/energiebilanzen/index.html. [Zugriff am 2307 2012].

2. Heimberger, M. (2015): Energy storage - optimisation of the placement and operation (in a Distribution Grid). Wien.

3. Jauschnegg, H., Rakos, C., Hackstock, R., Pfemeter, C., Kirchmeyr, F. (2013): Energiewende beim Heizen - Maßnahmen zu 55\% erneuerbarer Raumwärme, Erneuerbare Energie Österreich.

4. e-control (2016): STROMKENNZEICHNUNGSBERICHT 2016, Energie-Control Austria. Wien.

5. bmwfw; bmlfuw (2010): EnergieStrategie Österreich, Wien.

6. BMWFJ (2010): Nationaler Aktionsplan 2010 für erneuerbare Energie für Österreich (NREAP-AT).

7. Appelrath, H.-J., Lehnhoff, S., Rohjans, S., König, A. (2012): Hybridnetze für die Energiewende - Forschungsfragen aus Sicht der IKT, acatec MATERIALIEN, Berlin.

8. Agora Energiewende (2014): Stromspeicher in der Energiewende, Agora Energiewende, Berlin.

9. Kaufmann, T. (2016): Modellierung und Simulation von urbanen Stromversorgungsnetzen in einem multiskalaren Gesamtmodell, Wien: Dissertation an der TU Wien.

10. Groiß, C. (2013): Maximierung des regenerativen Erzeugungsanteils an de österreichischen Elektrizitätsversorgung, Wien: Dissertation an der TU Wien.

11. TU Wien ESE/EA, (2014): SYMBIOSE - Systemübergreifende optimale dezentrale Hybridspeicher, approbierter Endbericht zum Forschungsprojekt im Rahmen der 5. AS Neue Energien 2020, Wien.

12. JKU Linz - Energieinstitut, TU Wien - ESEA, MU Leoben - VTiU, MU Leoben EVT, (2016): Stadtwerke Leoben e.U., Smart Exergy Leoben - Publizierbarer Endbericht. 


\section{Autoren}

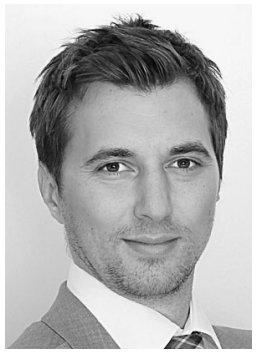

\section{Markus Heimberger}

wurde 1983 in Zwettl, Österreich, geboren Er studierte an der Technischen Universität Wien das Bachelorstudium Elektrotechnik. Anschließend absolvierte er das Masterstudium Energietechnik an der Technischen Universität Wien und Lunds Tekniska Högskola, Schweden. Am Institut für Energiesysteme und Elektrische Antriebe der TU Wien absolvierte er sein Doktoratsstudium, welches er 2016 mit Auszeichnung abschloss. Seine Doktorarbeit verfasste er zum Thema „Energy storage - optimisation of the placement and operation (in a Distribution Grid)". Seine Arbeitsschwerpunkte liegen im Bereich Hybridsysteme, Energieeffizienz und Gleichstromversorgung.

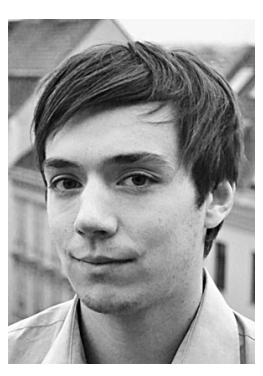

\section{Thomas Kaufmann}

wurde 1987 in Lilienfeld, Österreich, geboren. Er absolvierte das Bachelorstudium de Elektrotechnik und anschließend das Masterstudium im Studienzweig Energietechnik an der Technischen Universität Wien. Seit 2013 ist er am Institut für Energiesysteme und Elektrische Antriebe als Projektassistent tätig. Im Jahr 2016 schloss er mit der Doktorarbeit "Modellierung und Simulation von urbanen Stromversorgungsnetzen in einem multiskalaren Gesamtmodell", durchgeführt im Rahmen des Doktoratskollegs URBEM, sein Doktoratsstudium mit Auszeichnung ab. Seine Arbeitsschwerpunkte liegen im Bereich Hybridsysteme und Gleichstromnetze.

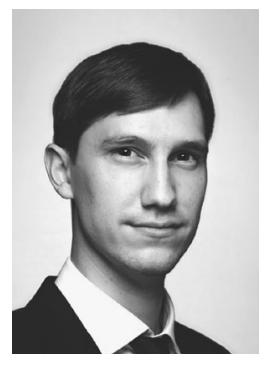

\section{Christoph Maier}

wurde 1984 in Wien, Österreich, geboren. Er studierte im Bachelorstudium Elektrotechnik an der Technischen Universität Wien und der University of Strathclyde, UK. Nach dem Abschluss begann der das Masterstudium der Energietechnik an der Technischen Universität Wien, welches er mit Auszeichnung abschloss. Am Institut für Energiesysteme und Elektrische Antriebe ist er seit 2012 als Projektassistent tätig und verfolgt dort sein Doktoratsstudium. Seine Arbeitsschwerpunkte liegen im Bereich konventionelle und nichtkonventionelle Energiespeicherung, Demand Side Management und Hybridsysteme.

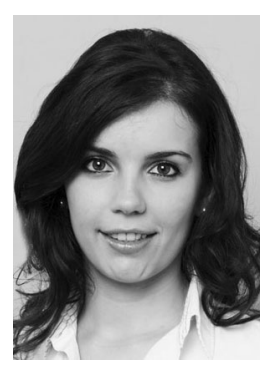

\section{Sabina Nemec-Begluk}

wurde 1985 in Sarajevo, Bosnien und Herzegowina, geboren. Sie absolvierte das Bachelorstudium der Elektrotechnik an der Technischen Universität Wien. Anschließend vertiefte sie sich im Rahmen ihres Masterstudiums an der Technischen Universität Wien im Bereich Energietechnik. Sie schloss ihr Masterstudium mit Auszeichnung ab. Am Institut für Energiesysteme und Elektrische Antriebe ist sie seit 2012 als Projektassistentin tätig und verfolgt seit 2012 ihr Doktoratsstudium. Ihre Arbeitsschwerpunkte liegen im Bereich Einbindung regenerativer Energiequellen in das bestehende Energiesystem, konventionelle und nichtkonventionelle Energiespeicherung und Hybridsysteme.

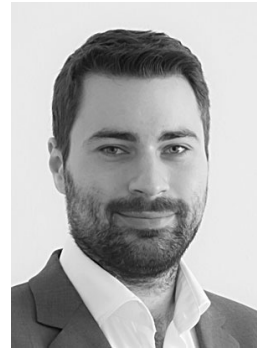

\section{Alexander Winter}

wurde 1987 in Wien geboren. Er absolvierte das Bachelorstudium der Elektrotechnik und anschließend das Masterstudium im Studienzweig Energietechnik an der Technischen Universität Wien. Seit 2014 ist er am Institut für Energiesysteme und Elektrische Antriebe als Projektassistent tätig. Seine Arbeitsschwerpunkte liegen im Bereich Hybridsysteme und Gleichstromnetze.

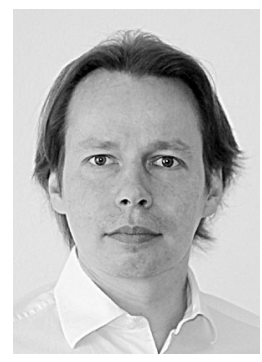

\section{Wolfgang Gawlik}

geboren 1971, studierte an der FriedrichAlexander-Universität (FAU) Erlangen-Nürnberg, Deutschland, Elektrotechnik mit Schwerpunkt Energietechnik. Nach Abschluss des Studiums 1997 war er zwei Jahre als Konstrukteur für Turbogeneratoren und stellvertretender Gruppenleiter Neukonstruktion bei der Siemens AG im Generatorenwerk Erfurt, Deutschland, tätig. Von 1999 bis 2004 war er Wissenschaftlicher Mitarbeiter und Wissenschaftlicher Assistent am Lehrstuhl für Elektrische Energieversorgung der FAU ErlangenNürnberg und promovierte dort 2004. Bis zu seiner Berufung als Universitätsprofessor für Energiesystemtechnik an der Technischen Universität Wien 2011 war er als Senior Key Expert System Dynamics und Projektmanager bei Siemens Power Technologies International, Erlangen, tätig. Seine Forschungsschwerpunkte umfassen Supergrids, Smart Grids/Microgrids und Elektromobilität, Universal Grids und Software zur Analyse, Planung und stationären und dynamischen Modellierung von Netzen. 